\title{
Grouping does not require attention
}

\author{
DOMINIQUE LAMY, HANNAH SEGAL, and LITAL RUDERMAN \\ Tel Aviv University, Tel Aviv, Israel
}

\begin{abstract}
Many theories of visual perception stipulate that Gestalt grouping occurs preattentively. Subjects' failure to report perceiving even salient grouping patterns under conditions of inattention challenges this assumption (see, e.g., Mack, Tang, Tuma, Kahn, \& Rock, 1992), but Moore and Egeth (1997) showed that although subjects are indeed unable to identify grouping patterns outside the focus of attention, effects of these patterns on visual perception can be observed when they are assessed using implicit, rather than explicit, measures. However, this finding, which is the only one to date demonstrating grouping effects without attention, is open to an alternative account. In the present study, we eliminated this confound and replicated Moore and Egeth's findings, using the Müller-Lyer illusion (Experiments 1 and 2). Moreover, we found converging evidence for these findings with a variant of the flanker task (Experiment 3), when the amount of available attentional resources was varied (Experiments 4 and 5). The results reinforce the idea that, although grouping outside the focus of attention cannot be the object of overt report, grouping processes can occur without attention.
\end{abstract}

Most models of visual perception assume that some basic perceptual processes are not contingent on capacity limitations for their execution and organize the visual field into units that form the basis for later, attentive processing (e.g., Neisser, 1967). Just what perceptual processes belong to this category has been the object of extensive research. In particular, whether or not Gestalt principles of organization occur preattentively has remained controversial for several years.

Some authors have argued that grouping processes require no attention (e.g., Duncan, 1984; for reviews, see Egeth \& Yantis, 1997; Lamy \& Tsal, 2001). Empirical evidence for this view comes from studies showing that segmentation of the visual field into perceptual groups imposes constraints on attentional selection, which is taken to indicate that grouping processes precede attentional processing. With divided-attention tasks, judging two attributes was shown to be easier when these belong to the same, rather than to different, perceptual groups (see, e.g., Baylis \& Driver, 1993; Behrmann, Zemel, \& Mozer, 1998; Duncan, 1984; Lavie \& Driver, 1996, Experiments 1-3; Treisman, Kahneman, \& Burkell, 1983; Vecera \& Farah, 1994; Watson \& Kramer, 1999). With Eriksen's flanker or response competition task (Eriksen \& Eriksen, 1974), distractors grouped with the target are typically more difficult to ignore than distractors that belong to a different perceptual group (e.g., Baylis \& Driver, 1992; Driver \& Baylis, 1989; Kramer \& Jacobson, 1991; but see Lamy \& Egeth, 2002, Experiment 4; Shomstein \& Yantis, 2002). Finally, in spatial-cuing tasks, subjects are faster

\footnotetext{
Support for this research was provided by the Israel Science Foundation, Grant 1382-04, to D.L. Correspondence concerning this article should be addressed to D. Lamy, Department of Psychology, Tel Aviv University, Ramat-Aviv, P.O. Box 39040, Tel Aviv 69978, Israel (e-mail: domi@post.tau.ac.il).
}

to redirect their attention within the same object than to another object (e.g., Abrams \& Law, 2000; Egly, Driver, \& Rafal, 1994; Lamy \& Egeth, 2002; Lamy \& Tsal, 2000; Moore, Yantis, \& Vaughan, 1998; Vecera, 1994). Because these findings show that attending to one part of an object entails that other parts of this object are also attended, they are held to demonstrate that attention selects objects that have been formed before selection has taken place.

However, we have claimed elsewhere (Egeth \& Lamy, 2003; Lamy \& Tsal, 2001) that findings supporting the object-based view of attentional selection do not necessarily imply, as some authors have argued (e.g., Yantis, 2000), that grouping does not require attention. Indeed, in all the studies surveyed above, at least one part of the relevant object (i.e., of the perceptual group for which objectbased effects were measured) was attended. As a result, it is conceivable that attending to an object part causes other parts of this object to be attended. To illustrate this point, consider the question of whether or not binding features into an object requires attention (e.g., Treisman \& Schmidt, 1982). Finding that illusory conjunctions occur outside the focus of attention does indicate that, without attention, no binding occurs. In contrast, finding that focusing attention on one feature of an object entails that its other features are also attended does not demonstrate that binding is preattentive but, rather, may indicate that it is the focusing of attention on one feature of an object that causes its other features to become bound together. Thus, a safer avenue by which to investigate whether or not grouping requires attention may be to measure grouping effects when the relevant perceptual group lies entirely outside the focus of attention.

The several studies in which the experimental procedure matched this requirement actually have supported the alternative view, according to which grouping does require attention. For instance, Ben-Av, Sagi, and Braun (1992) showed that subjects' performance in discriminat- 
ing between horizontal and vertical grouping, or in simply detecting the presence or absence of grouping in the display background, was severely impaired when attention was engaged in a demanding concurrent task-namely, identification of a target situated in the center of the screen. Mack, Rock, and their colleagues (Mack, Tang, Tuma, Kahn, \& Rock, 1992; Rock, Linnett, Grant, \& Mack, 1992) developed the inattention paradigm in order to determine what can be perceived with no attention to, and no intention to perceive, perceptual grouping. Mack et al. presented subjects with displays in which a cross appeared in the center of the screen, with a Gestalt grouping display as its surrounding background. The subjects had to judge which of the cross limbs was longer, so the background display was initially irrelevant to the task. But after a few trials, the observers were suddenly asked surprise questions about how the immediately preceding background display appeared to group. The basic finding, replicated in several studies (see Mack \& Rock, 1998, for a review), was that the observers were unable to report anything about grouping in the background display.

Moore and Egeth (1997) noted that the dependent measure in these studies (in those of Mack, Rock, and colleagues, as well as in Ben-Av et al., 1992) was the subjects' conscious report of grouping. They suggested that the fact that grouping cannot be overtly reported when attention is engaged in a demanding concurrent task does not necessarily imply that grouping requires attention. For instance, failure to report grouping may result from memorial failure. That is, grouping processes may occur preattentively, with grouping being perceived, yet not remembered. In order to test this possibility, these authors also used an implicit measure, instead of only an explicit measure, to assess whether or not grouping occurs outside the focus of attention. In their study, displays consisted of a matrix of uniformly scattered white dots on a gray background, in the center of which were two black horizontal lines. Some of the dots were black and were either randomly scattered (random-matrix trials) or grouped (pattern-matrix trials) to form the Müller-Lyer illusion (Experiment 3). The subjects attended to the two horizontal lines and reported which one was longer. These experiments started with a practice phase with only random-matrix trials, followed by an "illusion block" in which $50 \%$ of the trials were patternmatrix trials and the remainder were random-matrix trials. Responses were clearly influenced by the illusion. Therefore, the fact that elements lying entirely outside the focus of attention formed a group affected behavior, indicating that grouping does not require attention. The experiment ended with an "inattention block," the design of which was modeled after Mack et al.'s (1992). In this block, the subjects were tested on an explicit memory check (direct query and forced choice) that immediately followed a patternmatrix trial. The subjects were unable to remember or recognize the illusion patterns, despite the fact that their length judgments were again affected by the illusion. This result confirmed the authors' hypothesis that implicit measures may reveal that subjects perceive grouping, under conditions in which explicit measures do not.
Moore and Egeth's (1997) results are important because they constitute the only finding of grouping between object parts that lie entirely outside the focus of attention. As such, they appear to resolve the conflict surrounding the question of whether Gestalt principles of organization require attention. Or do they? A troublesome aspect of the stimuli used by Moore and Egeth is that both the target lines and the dots forming the illusion stimuli were black on a background of white dots, so that they formed a fairly strong group on the basis of their common color. It is, therefore, possible that attending to the black lines caused the black dots to become attended by virtue of their belonging to the same perceptual group as the attended lines; that is, grouping between the attended lines and the black dots, rather than grouping between the dots, might have caused the illusion to affect the judgment of length. In other words, with this procedure, Moore and Egeth may have failed to investigate grouping outside the focus of attention and, rather, may have examined grouping effects under conditions in which one of the object's parts (here, the black lines) was attended, as in the studies on objectbased selection reviewed above. If this turned out to be the case, the only finding to date showing grouping without attention would be disqualified.

Therefore, a decisive test for the claim that grouping is preattentive would be to conduct a replication of Moore and Egeth's (1997) study with lines and dots that differ in color. This was the objective of Experiments 1 and 2. They were similar to Moore and Egeth's third experiment, which took advantage of the Müller-Lyer illusion. Experiments 3-5 were designed to provide converging evidence, using a variant of the Eriksen response competition paradigm (Eriksen \& Eriksen, 1974).

\section{EXPERIMENT 1}

Several aspects of the Müller-Lyer stimuli have been manipulated in order to determine which factors are critical for obtaining the illusion. Line length and arrowhead angle (e.g., Dewar, 1967; Greene \& Nelson, 1997) and practice and exposure duration (Mountjoy, 1958), as well as hue (Pollack, 1970), have been found to affect the magnitude of the illusion. To our knowledge, the similarity in color between the shafts and the arrowheads has been manipulated in only one study (Mukerji, 1957), and despite great variability across subjects and color pairs, the general finding was that the illusion decreased when the colors of the shafts and the arrowheads differed, relative to when they were the same. The objective of Experiment 1 was to determine the extent to which the illusion would be reduced, if at all, when the lines and the dots differed in color, under conditions of full attention, and with parameters (e.g., stimuli, practice, and number of experimental trials) designed to be identical to those used in Moore and Egeth's (1997) study. Full attention to the dot pattern was obtained by explicitly drawing the subjects' attention to the fact that on each trial, arrowheads made up of colored dots appeared at the ends of the central lines. This preliminary experiment was necessary because it served as a baseline 
that would allow us to determine whether or not the potential finding that the illusion disappears in the different-color condition without attention can be interpreted as evidence that grouping requires attention. Indeed, the latter finding would become meaningless if a floor effect (i.e., the finding of no illusion) were to be observed with full attention.

\section{Method}

Subjects. The subjects were 10 Tel Aviv University undergraduates, who volunteered for the experiment. All had normal or correctedto-normal visual acuity and normal color vision.

Apparatus. Displays were generated by an Intel Pentium 4 computer attached to a 15 -in. TFT monitor, using $640 \times 480$ resolution graphics mode. Responses were collected via the computer keyboard. A chinrest was used to set viewing distance at $60 \mathrm{~cm}$ from the monitor.

Stimuli. The stimulus displays were similar to those used by Moore and Egeth (1997, Experiment 3) on pattern-matrix trials (see Figure 1). The fixation display was a black $0.6^{\circ} \times 0.6^{\circ}$ plus sign $(+)$ in the center of a gray background. Target displays included a matrix of white and colored dots, which was centered on the gray background, and two horizontally oriented colored line segments. The matrices were 28 columns $\times 22$ rows of dots. Each dot had a diameter of $0.40^{\circ}$. Dots within a row were separated by $0.64^{\circ}$ (center to center), and dots within a column were separated by $0.76^{\circ}$ (center to center). The line segments were centered from side to side within the matrix and were presented in its 7th and 16th rows, on top of the dots. Each of the two horizontal lines was equally likely to subtend either $5.00^{\circ}$ or $5.20^{\circ} .^{2}$ For trials in which one line segment was longer than the other, the longer segment was presented equally often in the top and bottom positions. On each trial, the colored dots in the matrix formed arrowheads, and the line segments formed shafts. Together, the line segments and dots formed figures similar to those used in the Müller-Lyer illusion. The heads pointed in on one shaft (hereafter, the wings-in shaft), whereas the heads pointed out on the other shaft (hereafter, the wings-out shaft). As is shown in Figure 1, even when the two lines were of the same length, the shaft of the wings-out display looked longer than that of the wings-in display. When the two lines were of different lengths, line lengths were either congruent or incongruent with the illusion; that is, the longer line was either the wings-out or the wings-in shaft, respectively. For half of the subjects, the central lines (or shafts) were black, and for the remaining half, they were red. Mask displays were matrices in which a random $30 \%$ of the dots were colored and the rest were white. Thus, the mask display did not include the line segments. A new mask was generated for each trial. For each subject, on same-color trials, the colored dots in the target display (i.e., the arrowheads) and in the mask were in the same color as the central lines, whereas on different-color trials, they were in the alternative color. The colors differed in luminance $\left(40 \mathrm{~cd} / \mathrm{m}^{2}, 21 \mathrm{~cd} / \mathrm{m}^{2}, 18 \mathrm{~cd} / \mathrm{m}^{2}\right.$, and $0 \mathrm{~cd} / \mathrm{m}^{2}$ for white, red, gray, and black, respectively, using a Minolta ColorCAL colorimeter light meter).

Procedure. The subjects were seated in a dimly lit room. Viewing distance was maintained at $60 \mathrm{~cm}$ from the computer monitor, using a chinrest. At the beginning of each session, the subjects were told that each trial would begin with a fixation plus sign, followed by a briefly presented stimulus containing two horizontal lines, at the ends of which arrowheads pointing either inward or outward and made up of colored dots would also appear. They were instructed that the lines would always differ in length. They were asked to maintain their gaze on the location of the fixation point and to report, after each trial, which of the two line segments appeared longer: the top or the bottom. They were instructed to use the numerical keypad and to press " 3 " if they judged the lower line to be longer and " 6 " if they judged the upper line to be longer. If they did not know which was longer, they were asked to guess. They were requested to be as accurate as possible and were informed that performance speed was not measured. The target display followed the fixation display after $1,500 \mathrm{msec}$; after $200 \mathrm{msec}$, it was replaced by the mask display, which remained on the screen until a response was given. The screen went blank for $500 \mathrm{msec}$ before the next trial began. Eye movements were not monitored, but the subjects were explicitly requested to maintain fixation throughout each trial.

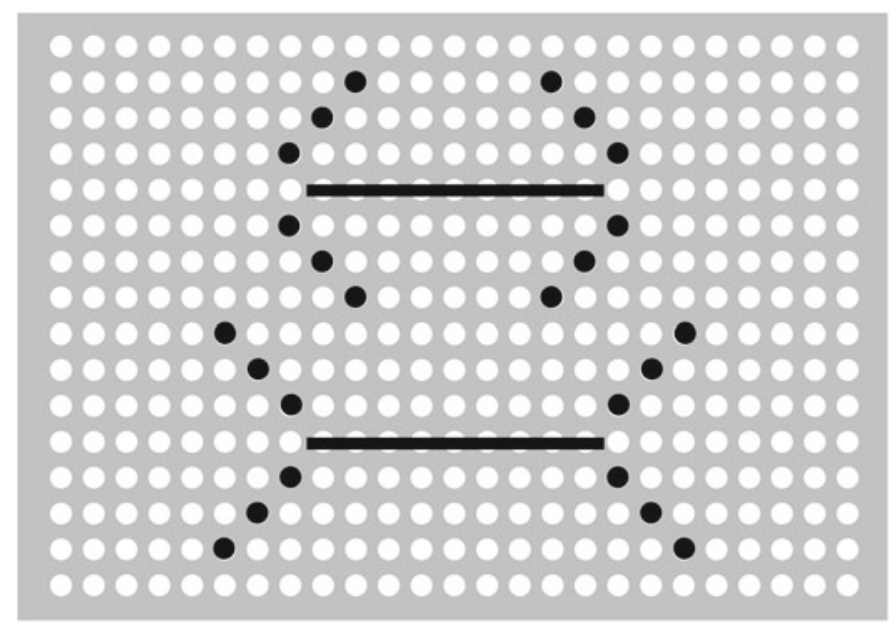

Figure 1. Stimulus display in Experiments 1 and 2. The example depicts the same-color pattern-matrix condition. The black dots formed arrowhead patterns. The two line segments for the length discrimination task were identical and were centered in the patterns in such a way that they formed the shafts for the arrowheads. The white dots were white, and the black dots were either red or black, as were the black line segments. Line segment color (red or black) was varied between subjects. Dot color (red or black) was varied within subjects in Experiment 1 and between subjects in Experiment 2. 
Conditions in which the lines were of the same versus a different length, the dots were of the same versus a different color, and the wings-out shaft was at the top versus the bottom were equally probable and randomly mixed. Thus, on different-length trials, illusioncongruent and -incongruent trials were equiprobable. The experiment began with one block of 10 practice trials, followed by one block of 32 experimental trials. No feedback was provided concerning the subjects' responses.

\section{Results}

Different-length trials. An ANOVA was conducted on accuracy scores in the different-length condition, with dot color (same vs. different) and illusion congruency (congruent vs. incongruent) as within-subjects factors and line color (red or black) as a between-subjects factor. The main effect of illusion congruency was significant, with higher accuracy scores on illusion-congruent than on illusion-incongruent trials [87.95\% and $35.23 \%$, respectively; $F(1,9)=29.87, p<.0003]$. The interaction between illusion congruency and dot color (same vs. different) did not reach significance $[F(1,9)=2.29, p>.1]$, despite a numerical trend toward a larger performance advantage on illusioncongruent than on illusion-incongruent trials when the lines and dots were in the same color (91.43\% vs. $28.26 \%$ ) than when they were in different colors $(85.42 \%$ vs. $42.86 \%$ ). Further analyses revealed that this advantage was significant in both conditions of dot color $[F(1,9)=$ $80.75, p<.0001$, and $F(1,9)=8.79, p<.02$, for same and different dot color, respectively]. There was no significant effect involving line length.

Same-length trials. Illusion scores were calculated as the percentage of trials in which the subjects judged the wings-out shaft to be longer than the wings-in shaft. That is, illusion scores significantly higher than $50 \%$ indicated that line length judgments were influenced by the Müller-
Lyer illusion (see Figure 2). An ANOVA conducted on same-length trials, with line color as a between-subjects factor and dot color as a within-subjects factor, revealed that the illusion was stronger when the lines and the dots were in the same color, rather than in different colors $[F(1,9)=8.79, p<.02]$. There was no significant effect involving line color. Further analyses showed that illusion scores were significantly above chance in both the samecolor $(87.06 \% ; z=6.63, p<.0001)$ and the differentcolor $(76.04 \% ; z=4.66, p<.0001)$ conditions.

\section{Discussion}

The results of Experiment 1 yielded three main findings. First, we observed a very robust effect of the illusion, using Moore and Egeth's (1997, Experiment 3, illusion block) stimuli and procedure under conditions of full attention. This effect was apparent not only with the standard illusion stimuli (same-length condition), but also in the fact that the subjects tended to mistakenly judge the wings-out shaft as being the longer one when it was actually the shorter one (different-length condition). Second, the early finding reported by Mukerji (1957) was replicated: The magnitude of the illusion decreased significantly when the shafts and arrowheads were in a different colors, rather than in the same color. Third, the illusion in the different-color condition remained well above 50\%that is, above the level that would be expected if the subjects had been guessing without bias.

These findings led us to conclude that conducting a replication of Moore and Egeth's (1997) study (Experiment 3, illusion block) - that is, the same experiment as Experiment 1 , but under conditions of inattention-should be informative despite the decrease in the illusion magnitude observed in the different-color condition. We reasoned

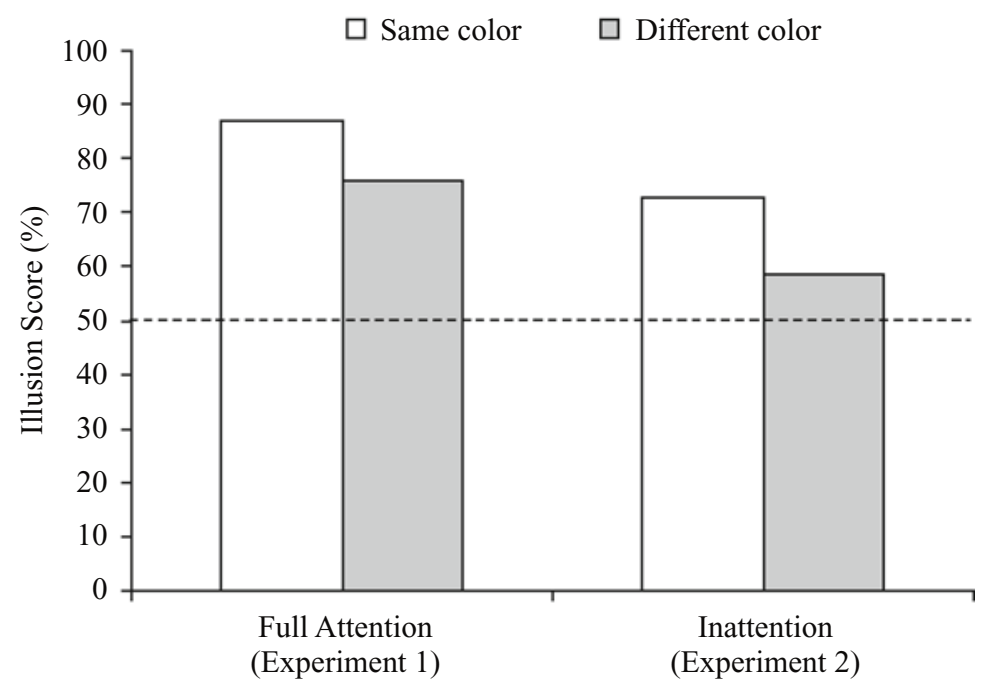

Figure 2. Results of Experiments 1 and 2. Mean illusion scores (percentage of trials in which the subjects judged the wings-out shaft to be longer than the wings-in shaft) on same-length trials in Experiment 1 and on pattern-matrix trials in Experiment 2, when the colors of the shafts and the arrowheads were the same versus when they were different. 
as follows. If grouping between the lines and dots was the critical factor for the illusion to be observed in Moore and Egeth's study, the decrement in the magnitude of the illusion in the different-color condition, relative to the same-color condition, should be substantially larger under conditions of inattention than the decrement that was observed in Experiment 1, under conditions of full attention. Indeed, when the whole display is attended (full-attention condition), the dots forming the arrowheads benefit from at least diffuse or distributed attention, which as Mack et al. (1992) noted, may suffice for grouping to occur. In contrast, when only the shafts are attended (inattention condition), if the dots are not attended as a consequence of their being grouped by common color with the attended the shafts, no illusion should be observed in the differentcolor condition, and illusion scores should be at chance level. On the contrary, if, as was intended by Moore and Egeth, grouping between the dots was the critical factor, the decrement in the magnitude of the illusion should remain roughly constant under conditions of attention versus inattention. Indeed, whether or not the shafts and the dots share the same color should not affect grouping between the dots, since in both cases, all the dots had the same color.

Moore and Egeth (1997) reported high illusion scores in the inattention condition with same-color shafts and dots (roughly $77 \%-88 \%$ ). Since we reported an average illusion score of $87 \%$ with similar stimuli in the full-attention condition, we should not expect the magnitude of the illusion to drop dramatically or disappear in the replication of our first experiment under conditions of inattention. It follows that, if grouping between the dots was the critical factor, illusion scores above chance level should be observed with shafts and dots differing in color, under conditions of inattention. In summary, despite the effect of same versus different color that was observed, the results of Experiment 1 eliminated our initial concerns regarding potential floor effects.

\section{EXPERIMENT 2}

Experiment 2 was the same as Experiment 1, except that, as in Moore and Egeth's (1997) illusion block, half of the pattern-matrix trials used in Experiment 1 were replaced by random-matrix trials and the subjects were provided with no information concerning the background surrounding the relevant lines. Thus, as did Moore and Egeth, we expected that the subjects would not pay attention to the fact that the colored dots formed arrowheads on part of the trials. The subjects were questioned immediately after the last trial of the experimental block, in order to determine whether or not they could report what patterns had appeared in the background.

\footnotetext{
Method

Subjects. The subjects were 16 Tel Aviv University undergraduates, who volunteered for the experiment. All had normal or correctedto-normal visual acuity and normal color vision.
}

Apparatus, Stimuli, and Procedure. The apparatus, stimuli, and procedure were the same as those in Experiment 1, except for the following changes. Random-matrix trials replaced the different-length pattern-matrix trials used in Experiment 1. On random-matrix trials, a random $8 \%$ of the dots were colored, and the rest were white. On pattern-matrix trials, the two lines were always of the same length, whereas on random-matrix trials, they always differed in length. The same versus different dot color variable was manipulated between, instead of within, subjects. Thus, there were four groups of subjects (red lines and dots, black lines and dots, black lines and red dots, and red lines and black dots). Again, the subjects were instructed to report which of two horizontal lines was longer, but they were not informed of the different types of trials (random- vs. pattern-matrix trials). That is, they were not told that the horizontal lines would sometimes be surrounded by arrowheads, and were not shown any examples of the stimuli. The practice block included 10 trials, all randomly drawn from the random-matrix condition. The subjects were run on one block of 32 experimental trials consisting of 16 random-matrix trials ( 8 trials with the longer line in the upper position and 8 trials with the longer line in the lower position) and 16 pattern-matrix trials ( 8 trials with the wings-out shaft in the upper position and 8 trials with the wings-out shaft in the lower position).

Immediately after the last trial of the experimental block, the subjects were asked three questions: direct query, forced choice, and confidence rating. The direct query was, "Did you notice any stimuli or pattern surrounding the lines?" In the forced choice question, the subjects were presented with one of four displays (see Figure 3) and were asked which of the patterns present in each display had appeared in the experimental block of trials. For half the subjects, the pattern in each display was in the same color as the relevant lines in the experimental block, and for the remaining half, it was in the alternative color. The order in which the four possible patterns were presented was counterbalanced across subjects. Finally, the confidence rating referred to the answer given to the forced-choice question. The confidence rating was $1,2,3$, or 4 , where 1 meant $r e$ ally just guessing, 2 meant not at all sure, 3 meant somewhat sure, and 4 meant very sure.

\section{Results}

The mean percent correct for reporting which line segment was longer on random-matrix trials was $79.30 \%$ $( \pm 5.00 \%)$. An ANOVA conducted on pattern-matrix trials with line color and same versus different dot color as between-subjects factors revealed that the illusion was stronger when the lines and the dots were in the same color, rather than in different colors $[F(1,15)=5.67$, $p<.02]$. There was no significant effect involving line color. Further analyses showed that illusion scores were significantly above chance in both the same-color condition $(72.66 \% ; z=5.13, p<.0001)$ and the different-color condition $(58.59 \% ; z=1.94, p<.03)$. Illusion scores are presented in Figure 2.

In the direct query, 5 out of the 16 subjects did not notice that the central lines were at all surrounded with dots and 5 subjects noticed the dots but did not discern that they formed a pattern; 6 subjects noticed that there were shapes surrounding the lines but could not recall anything concerning those shapes. In the forced choice question, only $18.75 \%$ of the subjects (i.e., 3 subjects) chose the correct pattern - that is, no more than would be expected by chance ( $25 \%$, or 4 subjects). Two of the latter subjects reported a level of confidence of 3 , and one reported a level of 2 . The pattern of results did not notably change 

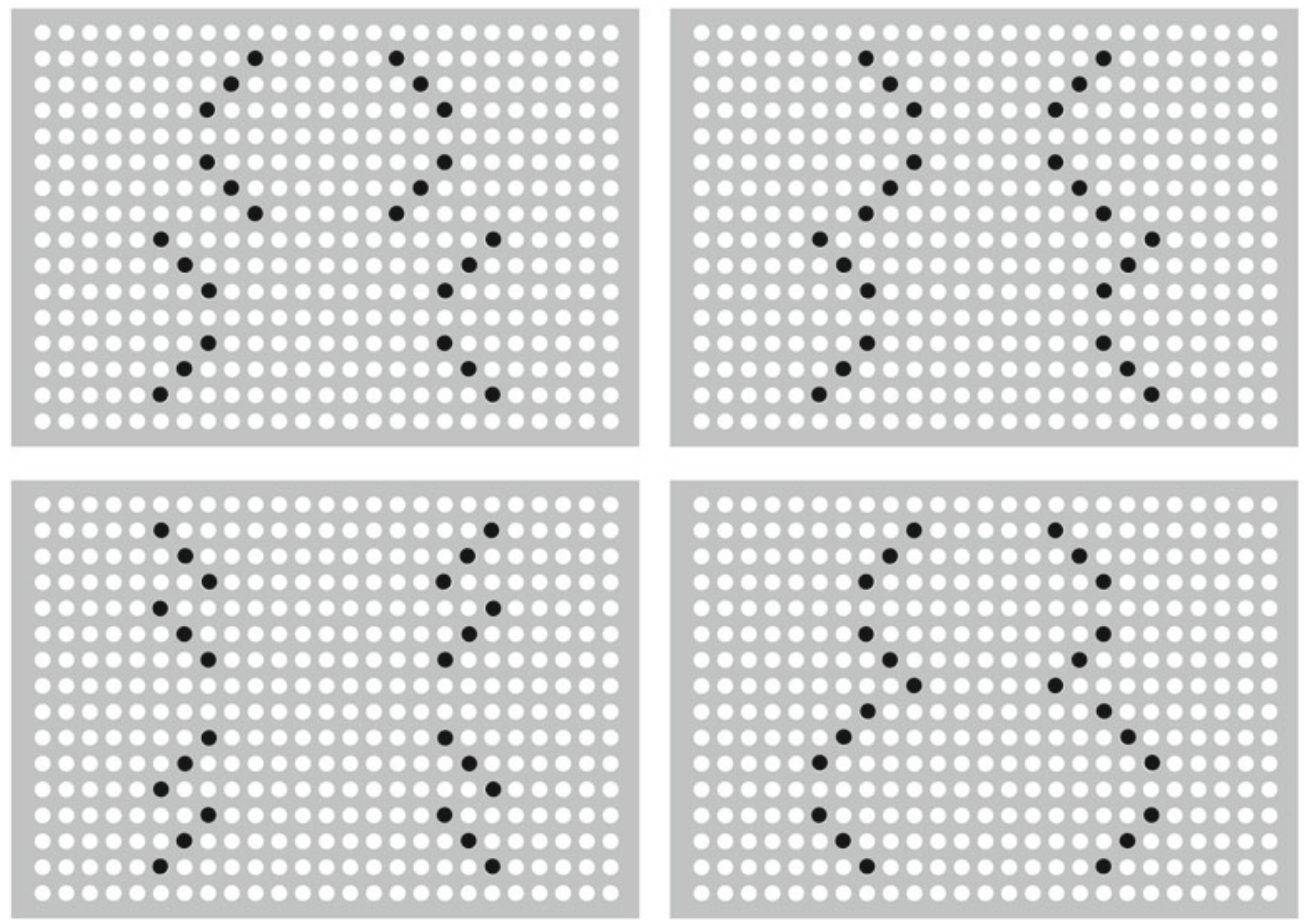

Figure 3. Experiment 2: Patterns presented in the forced choice query used to test the subjects' memory of the arrowhead patterns. Pattern presentation order was counterbalanced between subjects.

when these 3 subjects were excluded from analysis: Illusion scores remained significantly above chance in both the same-color condition $(70.54 \% ; z=4.65, p<.0001)$ and the different-color condition $(58.59 \% ; z=1.94, p<$ $.03)$, as well as significantly higher in the former condition than in the latter $[F(1,12)=3.85, p=.05]$.

\section{Discussion}

In both the same-color and the different-color conditions, responses were influenced by the Müller-Lyer pattern on pattern-matrix trials: The line segment that was part of the wings-out display was reported as longer than the line segment that was part of the wings-in display. Thus, Moore and Egeth's (1997) findings were replicated even when the relevant lines and the irrelevant dots were not grouped by common color. Moreover, the decrement in the magnitude of the illusion in the different-color condition, relative to the same-color condition, was virtually identical, in the present experiment under conditions of inattention, to that observed in Experiment 1 under conditions of full attention ( $14 \%$ [Cohen's $d=0.295$ ] and $11 \%$, [Cohen's $d=0.284$ ], respectively), as would be expected if grouping between the dots, rather than only grouping between the lines and the dots, was the critical factor for obtaining the illusion. These findings suggest that grouping by common color did occur under conditions of inattention. Finally, despite evidence of grouping on patternmatrix trials, the subjects were poor at reporting what those patterns were when they were asked about them directly, immediately after the last experimental trial.
It may be noteworthy that the lines and dots were also grouped by proximity. It remains possible, therefore, that the relatively weak illusion effect observed in the different-color condition might have resulted from grouping by proximity and might disappear if such grouping was eliminated. Grouping by proximity, however, is an inherent feature of the Müller-Lyer illusion and, as such, cannot be eliminated. It was therefore necessary to find a different procedure to measure grouping under conditions of inattention. This procedure would have to fulfill two constraints: It should not require explicit report of grouping (implicit measure), and it should include displays in which the attended, task-relevant objects are utterly ungrouped with the unattended elements, relative to which grouping is being investigated. The procedure used in Experiment 3 fulfilled both constraints.

\section{EXPERIMENT 3}

Experiment 3 was conducted in order to eliminate the potential confound of grouping by proximity, which could provide an alternative account for the results of Experiment 2 , and in order to provide a converging measure of grouping under conditions of inattention. We used a variant of the Eriksen response competition paradigm (Eriksen \& Eriksen, 1974). The subjects made a discrimination response to a target letter that appeared in the middle of the screen against the background of a matrix made up of white outline squares. As in Experiment 2, some of the squares were colored; on half of the trials, the col- 
ored squares were randomly scattered (random-matrix trials), and on the remaining half of the trials, they formed a pattern (pattern-matrix trials). This pattern formed a letter that elicited a response that was either compatible or incompatible with the response elicited by the target letter (compatible vs. incompatible trials, respectively). The colored squares and the target letter always differed in color and were separated by a distance of at least $3.5^{\circ}$ of visual angle. Thus, they were not grouped in any way. We expected that if grouping by common color occurs outside the focus of attention, the colored squares would be perceived as forming a letter, and compatibility effects would be observed - that is, shorter response latencies on compatible than on incompatible trials. Moreover, since exposure times and percentage of pattern-matrix trials were the same as in Moore and Egeth's (1997) study (as well as in Experiment 2), we also expected chance performance in the subjects' explicit reports of what patterns had appeared in the background.

\begin{abstract}
Method
Subjects. The subjects were 10 Tel Aviv University undergraduates, who participated in the experiment for course credit. All had normal or corrected-to-normal visual acuity and normal color vision.

Apparatus and Stimuli. The apparatus was the same as that in Experiment 1. Target displays consisted of a matrix of 15 columns $\times$ 15 rows of squares, each subtending $0.5^{\circ}$ on a side, and of one target letter (see Figure 4). The squares were separated from each other by $1^{\circ}$ horizontally and vertically. The target letter was created by removing one line segment from the outline square that appeared at the center of the matrix (8th column and 8th row). Thus, the target letter was a $C$, a horizontally inverted $C$, a $U$, or a vertically inverted $U$. In the red-target group ( $50 \%$ of the subjects), the target letter was red on each trial, and in the green-target group ( $50 \%$ of the subjects), it was green. On random-matrix trials, a random $6 \%$ of the squares were colored, and the rest were white. On pattern-matrix trials, the colored squares made up a letter shape (the distractor), similar to Navon's (1977) global letters. Each possible distractor was a global
\end{abstract}

letter made up of a total of 13 local squares (5 local squares on a side, with one missing side), so that the possible distractor forms were identical to the possible target letter forms. The distractor and the target letter were centered on each other, so that the distractor surrounded the target letter. Thus, the nearest colored square was distant from the target letter by $3.5^{\circ}$ of visual angle, center to center. Mask displays were matrices in which a random $30 \%$ of the outline squares were colored and the rest were white. The colored squares in the random-matrix, pattern-matrix, and mask displays always differed from the target letter in color. Thus, they were always green in the red-target group and red in the green-target group. The background was black.

Again, immediately after the last experimental trial, the subjects were asked surprise questions. The direct query was, "Did you notice any stimuli or pattern surrounding the target letter?" The subjects were then presented with one of the patterns used in the experiment and were asked whether or not they had seen this pattern during the experimental block.

Procedure. The subjects' task was to respond to the target letter shape. They were requested to press " 1 " with their left index finger on the computer keyboard if the target letter was a $C^{\prime}$ or an inverted $C$ and " 2 " with their right index finger if it was a $U$ or an inverted $U$. They were asked to respond as quickly as possible, while maintaining high accuracy. This procedure created two conditions of target-distractor response compatibility, in that the target and the distractor elicited either the same response (compatible trials) or opposite responses (incompatible trials). Target-to-response mapping was counterbalanced between subjects. The subjects were not informed of the different types of trials (random- vs. pattern-matrix trials).

The target display appeared for $200 \mathrm{msec}$ and was replaced by the mask display, which remained on the screen until a response was given. The screen went blank for $500 \mathrm{msec}$ before the next trial began. Error trials were followed by a $500-\mathrm{msec}$ feedback beep. Eye movements were not monitored, but the subjects were explicitly requested to maintain fixation throughout each trial.

Design. The design included two within-subjects variables of matrix type (random vs. pattern) and target-distractor compatibility (compatible vs. incompatible) and one between-subjects variable of target letter color. Conditions of matrix type, target shape, and distractor shape were equally probable and randomly mixed. Thus, on
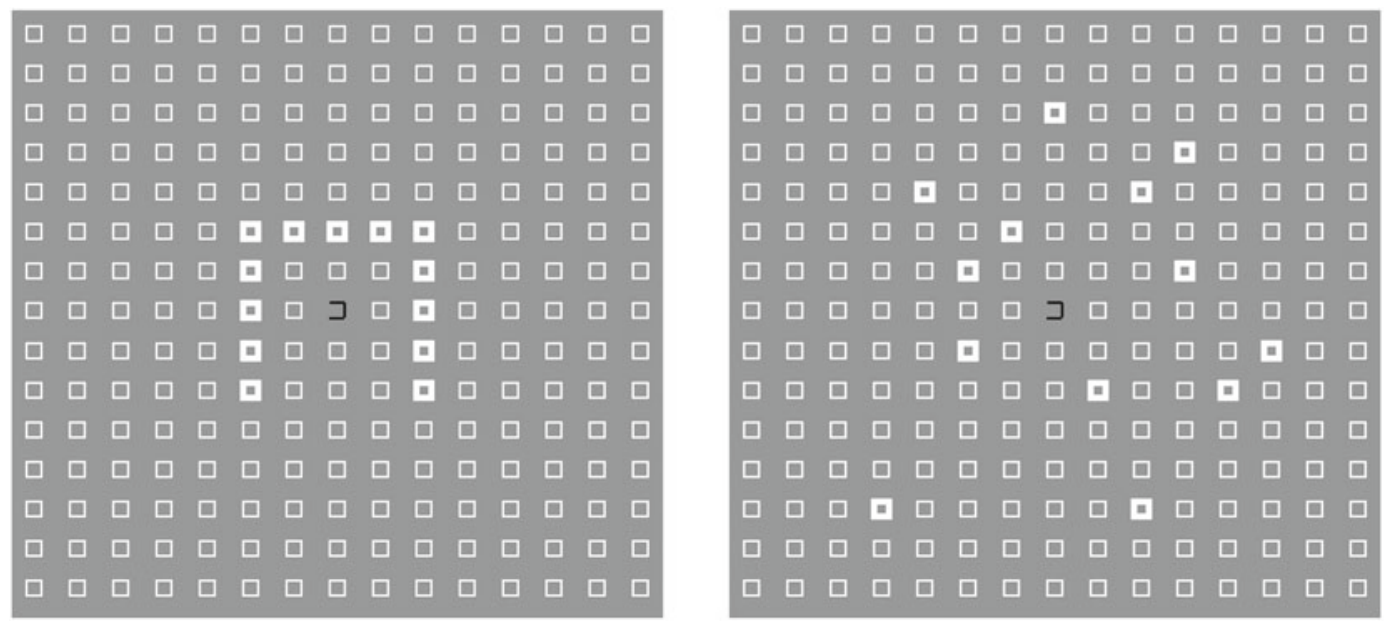

Figure 4. Stimulus display in Experiment 3. Left panel: The example depicts a pattern-matrix trial in which the colored squares formed a shape that elicited an incompatible response. Right panel: The example depicts a random-matrix trial. The thin white outline squares were white, and the thick white outline squares were red for half the subjects and green for the other half. The black central target letter always differed in color from the thick outline squares and was either red or green. 
pattern-matrix trials, the target and the distractor were equally likely to be compatible or incompatible. The experiment began with one block of 50 practice trials, all randomly drawn from the randommatrix condition. The practice block was followed by 720 experimental trials, divided into six blocks of 120 trials each. The subjects were allowed a rest period after each block.

\section{Results}

When prompted after the last experimental trial, only 3 of the subjects reported noticing that some of the background squares had differed in color from the other squares, and none reported that these squares had formed a pattern. When shown one letter pattern that had appeared during the experiment, all the subjects responded that no such pattern had been in the background.

The data from 1 subject were excluded from analysis because this subject made more than $20 \%$ errors. In all reaction time (RT) analyses, error trials $(5.0 \%$ of all the trials) were removed from analysis, and the RTs for each subject were sorted into cells according to conditions of matrix type and target-distractor compatibility. RTs exceeding the mean of that cell by more than 3.5 standard deviations were trimmed. This removed fewer than $1 \%$ of all observations.

An ANOVA on the RT data, with matrix type and target color as factors, revealed that the subjects were faster to respond on pattern- than on random-matrix trials [380 vs. $408 \mathrm{msec}$, respectively; $F(1,8)=6.74, p<.04] .{ }^{3}$ No effect involving target color approached significance. The same analysis on the accuracy data revealed no significant effect $(F \mathrm{~s}<1)$.

A separate ANOVA was conducted on pattern-matrix trials with target color and target-distractor compatibility as factors. The subjects were faster to respond on compatible than on incompatible trials [ $373 \mathrm{vs.} 386 \mathrm{msec}$, respectively; $F(1,8)=10.13, p<.02]$. Again, no effect involving target color approached significance. The same analysis on the accuracy data revealed no significant effect $\left(F_{\mathrm{S}}<1\right)$.

\section{Discussion}

The results show that the shape of a perceptual group lying entirely outside the focus of attention and not grouped with the attended target affected the subjects' responses. To have produced compatibility effects, the colored squares must have been grouped to form the letter patterns. As in Experiment 2, despite the fact that evidence of grouping was shown for the pattern-matrix trials, the subjects were unable to report what those patterns had been when they were asked about them directly after the last experimental trial and even found it difficult to believe that letter patterns had indeed been presented to them. This finding replicates the dissociation reported by Moore and Egeth (1997) between explicit and implicit measures of grouping perception: Although the subjects failed to recognize the unattended perceptual groups, these perceptual groups affected their performance.

Of course, the fact that the subjects were unable to remember the patterns does not ensure that these were not attended. This concern cannot be resolved by minimizing demands on memory (for instance, by asking the subjects to report on the pattern immediately after each trial). Indeed, an inability to report on the pattern does not entail that this pattern was unattended but, even with very short retention intervals, might still be a result of memory failure. Accordingly, although the subjects failed to notice the patterns in the background, and although the task required attention to be tightly focused on the center of the screen, it remains possible that, on some trials, the subjects spread their attention on the whole display or even inadvertently directed their attention to the periphery. Thus, the observed illusion (Experiment 2) and compatibility (Experiment 3) effects may, in fact, have resulted from just a few trials in which the subjects allocated some attention to the pattern surrounding the target lines or letter. In order to test this alternative account, we analyzed the RT distributions obtained in Experiment 3, so that the individual distributions for each subject and compatibility condition were averaged with the Vincentizing procedure (Ratcliff, 1979). If grouping affected performance only on a small portion of the trials in which the peripheral stimulus was inadvertently attended, then it would be expected that for a substantial part of the RT distribution, RTs in the compatible and the incompatible conditions should be similar (with RTs on compatible trials being equally likely to be shorter or longer than RTs on incompatible trials). In contrast, if grouping outside the focus of attention affected performance, RTs should be shorter on compatible than on incompatible trials throughout the RT distribution. ${ }^{4}$ As is clear from Figure 5, the data support the latter prediction.

\section{EXPERIMENT 4}

The objective of Experiment 4 was to further examine the possibility that the results obtained in Experiment 3 might have stemmed from some leakage of attention to the peripheral patterns. The finding of shorter RTs on compatible than on incompatible trials throughout the RT distribution (Figure 5) is inconsistent with the claim that the subjects occasionally attended to the peripheral stimuli. However, it remains possible that some attention accrued to the peripheral stimuli throughout the experiment. Lavie and Tsal (1994) proposed that perceptual load of relevant information determines the extent to which irrelevant information is processed. In support of this claim, Lavie (1995) showed that response compatibility effects are obtained only under low-load conditions and disappear under high-load conditions. The shape discrimination task used in Experiment 3 was relatively easy, and it is therefore conceivable that spare attentional capacities might have accrued to the peripheral stimuli. If this happened to be the case, our finding that grouping of irrelevant peripheral stimuli affects performance would not demonstrate that grouping can proceed without attention. The objective of Experiment 4 was to test this possibility. To do so, we manipulated the level of central task difficulty. ${ }^{5}$ If grouping requires some attention, compatibility 


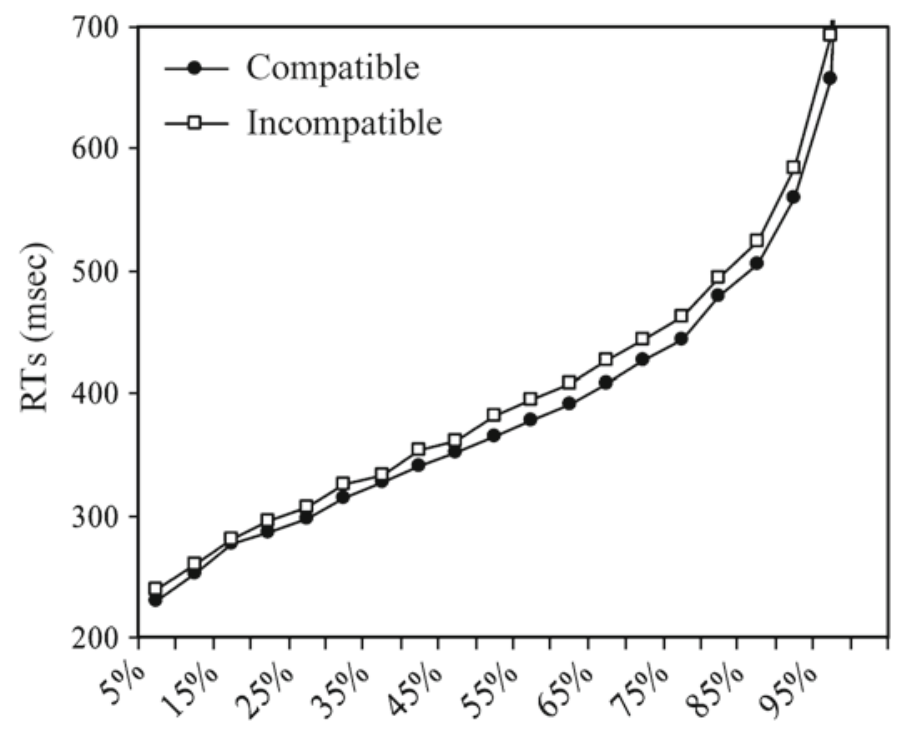

Figure 5. Results of Experiment 3. Vincentized reaction time (RT) distributions for pattern-matrix trials in the compatible versus incompatible conditions.

effects should be larger when the central task is easy than when it is difficult. Indeed, in the condition in which the central task is difficult, more attention should be absorbed by it, thus leaving less attention available for grouping to occur. Experiment 4 was identical to Experiment 1, except for the fact that level of difficulty of the central task was manipulated.

\section{Method \\ Subjects. The subjects were 12 Tel Aviv University undergradu- ates, who participated in the experiment for course credit. All had normal or corrected-to-normal visual acuity and normal color vision. \\ Apparatus, Stimuli, Procedure, and Design. The apparatus, stimuli, procedure, and design were identical to those in Experi- ment 3, except for the following changes. There were two conditions of central task difficulty. In the easy condition, the central task was the same as that in Experiment 3. In the difficult condition, the target stimulus was drawn in a broken, instead of an unbroken, line. Each side of the target letter consisted of three $0.12^{\circ}$-long dashes, separated by $0.07^{\circ}$ from each other. Each subject was run on both conditions of central task difficulty, each of which consisted of 50 practice trials, all randomly drawn from the random-matrix condition, and three blocks of 120 experimental trials each. At the end of the experiment, the subjects were asked the following three questions. (1) Did you notice that a differently colored square in the background formed a pattern around the target letter? (2) Did you see a square pattern with a missing side, similar to the target? (3) How positive are you (5 = absolutely sure, down to $1=$ not sure at all $)$ ?}

\section{Results}

When prompted after the last experimental trial, 2 of the subjects reported seeing patterns in the background and correctly described them with a high degree of confidence (levels 4 and 5). The remaining subjects reported noticing no pattern and claimed not to have seen any pattern similar to the target shape, with high degrees of con- fidence (7 subjects with level 5, 2 subjects with level 4, and 1 subject with level 3 ).

The data from 1 subject were excluded from analysis because this subject made more than $20 \%$ errors. The data from the 2 subjects who reported seeing the patterns in the background were also excluded. ${ }^{6}$ In all RT analyses, error trials (5.6\% of all the trials) were removed from analysis, and RTs for each subject were sorted into cells according to conditions of matrix type, target-distractor compatibility, and central task difficulty. RTs exceeding the mean of that cell by more than 3.5 standard deviations were trimmed. This removed fewer than $1 \%$ of all observations. Preliminary analyses showed no effect involving target letter color. The data were, therefore, collapsed across conditions of target letter color.

An ANOVA on the RT data, with matrix type and central task difficulty as factors, revealed a main effect of central task difficulty $[F(1,10)=10.49, p<.02]$. No effect involving matrix type reached significance. The same analysis on the accuracy data revealed no main effect of either central task difficulty or matrix type $[F(1,8)=$ 2.36, $p>.1$, and $F<1$, respectively] and no interaction between these factors $(\mathrm{F}<1)$.

A separate ANOVA was conducted on pattern-matrix trials, with central task difficulty and target-distractor compatibility as factors. Mean RTs are shown in Figure 6. The main effect of central task difficulty was significant, with shorter RTs in the easy condition $[F(1,8)=7.99, p<$ .03]. The main effect of compatibility was also significant, with shorter RTs on compatible than on incompatible trials $[F(1,8)=24.51, p<.002]$. This effect, however, was not modulated by central task difficulty $[F(1,8)=1.14$, $p>.3$, with comparable effects of compatibility in the 

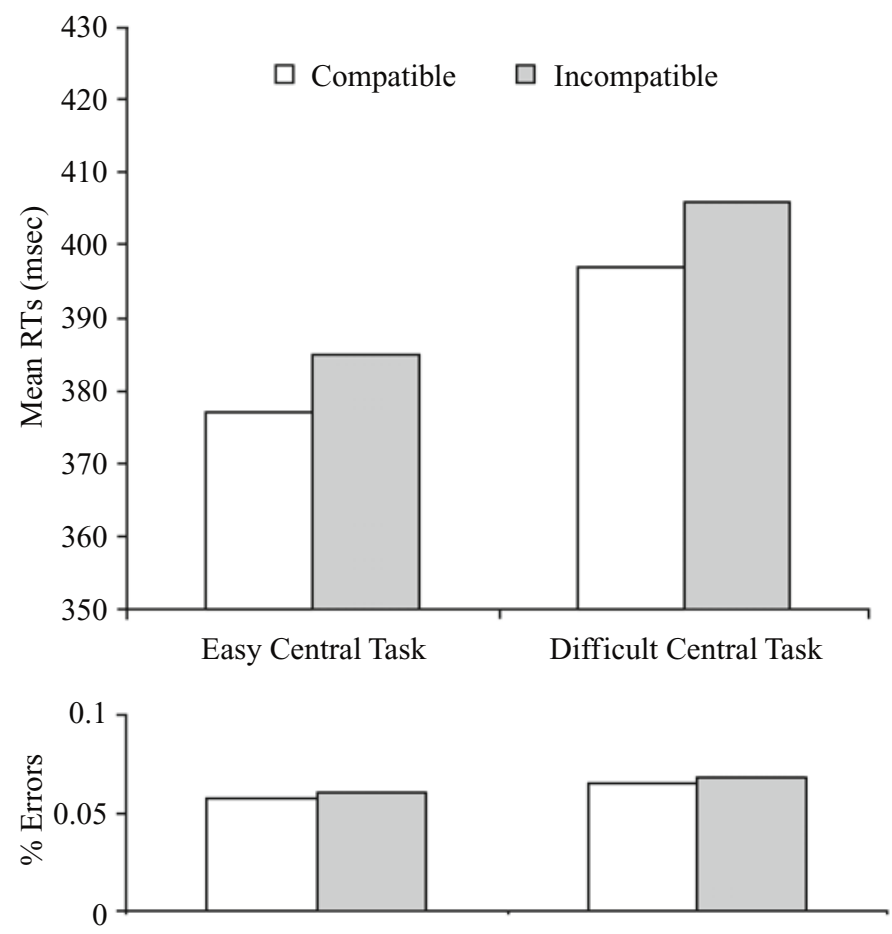

Figure 6. Results of Experiment 4. Mean reaction times (RTs, in milliseconds) as a function of the response elicited by the peripheral distractor (compatible vs. incompatible) and central task difficulty (easy vs. difficult). Central task difficulty was manipulated by drawing the central target either with a continuous line (easy condition) or with a broken line (difficult condition).

easy and the difficult conditions ( 8 and $9 \mathrm{msec}$, respectively). Although the compatibility effect was quantitatively small, it is noteworthy that it was observed in all 9 subjects. The same analysis on the accuracy data revealed no main effect of either central task difficulty or targetdistractor compatibility $[F(1,8)=1.42, p>.2$, and $F<$ 1 , respectively] and no interaction between these factors $(F<1)$.

\section{Discussion}

The fact that RTs were significantly longer in the difficult than in the easy condition confirms that the manipulation of difficulty level was successful. If grouping required attention, compatibility effects should have been larger when the central task was easy than when it was difficult. Yet compatibility effects of the same magnitude were observed in the two conditions of task difficulty. The results of Experiment 4 thus suggest that the compatibility effects observed in Experiment 3 did not result from spare attentional resources being allocated to the peripheral groupings.

In several studies, procedures similar to ours have been used to manipulate perceptual load. We manipulated difficulty of target discrimination by drawing the target in a broken versus unbroken line. In the same vein, Handy and Mangun (2000; see also Handy, Soltani, \& Mangun, 2001) varied target signal-to-noise ratio between load conditions by presenting targets unmasked in the low-load condition and masked in the high-load condition. They found that this manipulation modulated early ERP components and concluded that perceptual load mediates early selection. However, recent findings by Lavie and de Fockert (2003) warn against using degradation of the target stimulus as a manipulation of perceptual load. Following Norman and Bobrow's (1975) distinction, Lavie and de Fockert dissociated effects of data limits from effects of resource limits on distractor processing of irrelevant information. They showed that whereas increasing the number of irrelevant objects in the display (i.e., increasing resource limits) eliminated distractor interference, degrading the sensory quality of the target by reducing contrast, size, presentation duration, or retinal acuity (i.e., increasing data limits) in fact slightly increased distractor interference.

\section{EXPERIMENT 5}

Because the manipulation used in Experiment 4 clearly affected data limits, rather than resource limits, the fact that it did not reduce the magnitude of the compatibility effect produced by the global shape cannot be taken to indicate that grouping does not require attention. In order to address this concern, it would be necessary to use a manipulation of task difficulty that affects resource demands, rather than the quality of sensory input. In Ex- 
periment 5, instead of degrading the target, we increased target-distractor similarity (the target was always white; that is, it had the same color as the surrounding squares) and manipulated attentional load by varying distractor heterogeneity. In the easy (low-heterogeneity) condition, all the shapes immediately adjacent to the target were squares (as in Experiments 4 and 5), whereas in the difficult (highheterogeneity) condition, these squares were replaced by arrows in various orientations (see Figure 7). Increasing distractor heterogeneity at relatively high levels of targetdistractor similarity is known to increase demands on attentional capacity (Duncan \& Humphreys, 1989).

Note, however, that there may be an inherent problem with this experimental rationale. The finding that compatibility effects are eliminated under conditions of high perceptual load has been repeatedly reported with distractors for which shape discrimination did not require grouping (e.g., Lavie, 1995). Thus, should compatibility effects be eliminated with the present stimuli under high-load conditions, it would not be possible to ascribe such a finding unambiguously to the fact that attentional resources are necessary for grouping to occur. Indeed, it may, instead, be attributed to the fact that, in order to produce compatibility effects, any distractor must receive some attention (Lavie, 1995).

In order to circumvent this problem, we must compare the effects of spare attentional capacity when perceiving the shape of the irrelevant distractor requires grouping processes relative to when it does not. If grouping requires attention, then with low load, we should expect higher compatibility effects in the latter instance than in the former. Indeed, whereas all spare resources should contribute to increase interference from the irrelevant distractor when perceiving its shape does not require attention, part of these resources should be consumed by grouping

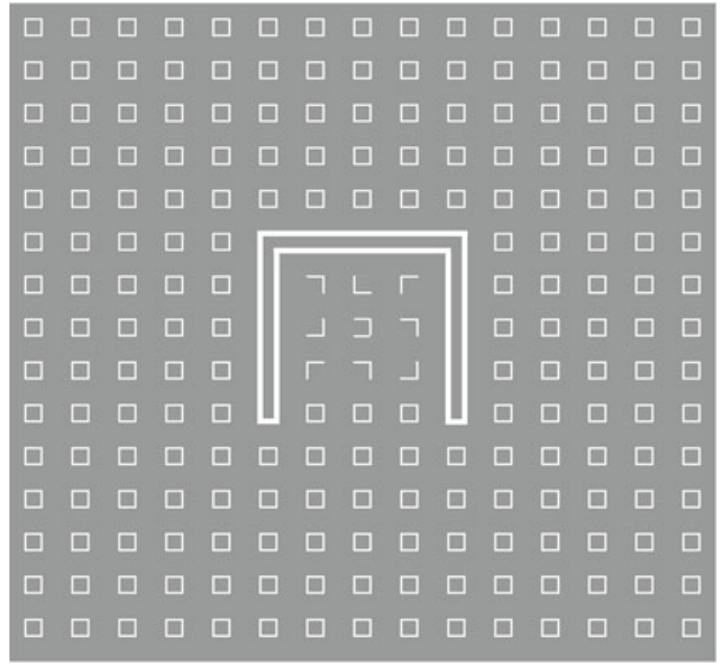

Figure 7. Stimulus display in Experiment 5. The example depicts an incompatible trial from the single uniformly connected condition with a difficult central task (high target-distractor heterogeneity). processes otherwise. Conversely, if grouping can proceed without attention, increasing the amount of available attention should produce the same interference whether or not perceiving the distractor's shape requires grouping.

Palmer and Rock (1994) distinguished between several hierarchically organized levels of representation, among them single uniformly connected (single-UC) regions, defined as connected regions with uniform visual properties, such as color or texture, and grouped uniformly connected (grouped-UC) regions, which are larger representations made up of single-UC regions grouped on the basis of Gestalt principles. According to their theory of perceptual organization, perceiving grouped-UC regions as unitary objects requires the operation of grouping processes, whereas perceiving single-UC regions does not. In Experiment 5, we relied on this distinction and manipulated the peripheral distractor surrounding the target accordingly. In the grouped-UC condition, the peripheral letter was identical to that in Experiment 4. In the single-UC condition, the peripheral letter was drawn in a continuous line, so that it formed an outline shape, the contour of which overlapped that of the peripheral letter in the grouped condition.

To summarize, then, Experiment 5 differed from Experiment 4 essentially in two respects: (1) Task difficulty was manipulated so as to affect resource, rather than data, limits, and (2) type of peripheral letter distractor was manipulated so as to create a condition in which perceiving the distractor shape did not require grouping (single-UC condition) and a condition in which it did require grouping (grouped-UC condition). In line with the perceptual load hypothesis (Lavie, 1995), we expected the peripheral letter distractor to produce little or no compatibility effects in the difficult condition in both the single-UC and the grouped-UC conditions. The critical prediction however, was that if grouping does not require attention, the spare attentional capacity available in the easy condition should result in the same amount of interference for both types of distractor shapes.

\section{Method}

Subjects. The subjects were 13 Tel Aviv University undergraduates, who participated in the experiment for course credit. All had normal or corrected-to-normal visual acuity and normal color vision.

Apparatus, Stimuli, Procedure, and Design. The apparatus, stimuli, procedure, and design were identical to those in Experiment 4 , except for the following changes. A stimulus example is shown in Figure 7. There were again two conditions of central task difficulty. The easy condition was the same as that in Experiment 4, except that the target shape was white - that is, it had the same color as the background squares making up the matrix. The difficult condition was chosen to be demanding enough to exhaust attentional resources. It was identical to the easy condition, except that for each of the squares immediately surrounding the target shape, two contiguous sides were erased. That is, the target was surrounded by arrows, with each arrow pointing in one of four possible directions, randomly. There were two conditions of type of distractor shape. In the grouped-UC condition, the distractor shape was identical to that in Experiment 4. In the single-UC condition, the distractor shape was drawn in a continuous line, so that it formed an outline shape, the contour of which overlapped that of the distractor shape in the grouped-UC condition. Note that the amount of sensory evidence 
(the number of colored segments) was identical in the two conditions. Each subject was run on each condition of distractor shape type, with order of condition of distractor shape type counterbalanced between subjects. Each condition of distractor type included one block in the easy central task condition and one block in the difficult task condition. For each subject, order of central task difficulty was the same for each condition of distractor type, and the order was counterbalanced between subjects. Each of the four blocks making up the experiment began with 20 practice trials, all randomly drawn from the random-matrix condition, followed by 180 experimental trials.

\section{Results}

When prompted after the last experimental trial, only 1 subject reported noticing patterns in the background and recognized them with a high degree of confidence. The remaining subjects did not notice the patterns and claimed not to have seen any pattern similar to the target shape, with high degrees of confidence.

The data from 1 subject were excluded from analysis because this subject made more than $20 \%$ errors. The data from the subject who reported seeing the patterns in the background were also excluded. ${ }^{7}$ In all the RT analyses, error trials (3.8\% of all the trials) were removed from analysis, and the RTs for each subject were sorted into cells according to conditions of matrix type, target-distractor compatibility, central task difficulty, and distractor shape type. RTs exceeding the mean of that cell by more than 3.5 standard deviations were trimmed. This removed fewer than $1 \%$ of all observations.

An ANOVA on the RT data, with matrix type and central task difficulty as factors, revealed a main effect of central task difficulty $[F(1,10)=10.75, p<.009]$. No effect involving matrix type approached significance $(F \mathrm{~s}<1)$. The same analysis on the accuracy data revealed no significant effect (all $F$ s $<1$ ).

A separate ANOVA was conducted on pattern-matrix trials, with distractor shape type (grouped-UC vs. singleUC), central task difficulty (easy vs. difficult), and targetdistractor compatibility (compatible vs. incompatible) as factors. Mean RTs are shown in Figure 8. The main effect of central task difficulty was significant, with shorter RTs in the easy condition $[F(1,10)=8.54, p<.02]$. The interaction between compatibility and central task difficulty was significant $[F(1,10)=6.14, p<.04]$. Planned contrasts revealed that there was a significant compatibility effect in the easy condition $[F(1,10)=10.60, p<.009]$ and none in the difficult condition $(F<1)$. Notably, this interaction was not modulated by type of distractor shape $(F<1)$. That is, the compatibility effect did not differ between the single-UC and the grouped-UC conditions in either the easy or the difficult condition $(F \mathrm{~s}<1)$. As further evidence that compatibility effects were not modulated by type of distractor shape (i.e., by whether or not perceiving the distractor shape required grouping), note that out of 11 subjects, 9 versus 8 subjects in the singleand the grouped-UC conditions, respectively, showed a compatibility effect in the expected direction in the easy condition, and 5 versus 6 subjects, respectively, in the dif- ficult condition. The same analysis on the accuracy data revealed no significant effect (all $F \mathrm{~s}<1$ ).

\section{Discussion}

Again, the fact that RTs were significantly longer in the difficult than in the easy condition confirms that the manipulation of difficulty level was successful. In contrast with the manipulation used in Experiment 4, which affected data limits, in the present experiment distractor heterogeneity was varied, which is known to affect resource limits (Duncan \& Humphreys, 1989). Increasing task difficulty by increasing resource limits eliminated distractor interference (Experiment 5), whereas increasing data limits by degrading the sensory quality of the target did not decrease distractor interference (Experiment 4). Thus, the results reported by Lavie and de Fockert (2003) were replicated. The critical finding concerns the comparison between the grouped-UC and the single-UC conditions. Our rationale was the following. It is not possible to assess whether or not grouping requires attention by measuring the effects of an irrelevant perceptual group under conditions in which no attention at all accrues to this perceptual group, because no compatibility effects should be expected, even if grouping processes are not required (Lavie, 1995). Conversely, however, if the irrelevant perceptual group produces compatibility effects when it receives some attention, this might be attributed to the fact that compatibility effects from any distractor are contingent on attention, but it might also be attributed to the fact that grouping itself is contingent on the availability of attentional resources. In order to solve this problem, we introduced a baseline condition, in which the surrounding letter was a single-UC region and, thus, did not require grouping in order to be perceived. We reasoned that in line with the perceptual load hypothesis, substantially increasing attentional demands should eliminate compatibility effects for both the single-UC and the grouped-UC distractors, whether or not grouping requires attention. We expected the critical answer to emerge from the pattern of results in the easy condition. If grouping requires attention, more resources should be used up in the grouped-UC condition, in which an extra attention-consuming step, grouping, is necessary for shape perception, relative to the single-UC condition. Yet compatibility effects of the same magnitude were observed in the grouped-UC and the single-UC conditions, suggesting that the same amount of attentional resources was necessary in the two conditions and that grouping therefore requires no attention.

Taken together, the results of Experiments 3-5 confirm those observed in Experiment 2, with no possible concern that (1) attending to the target letter caused the surrounding perceptual group to be attended or that (2) occasional attentional leakages or (3) accrual of residual attentional resources to the periphery allowed grouping to occur. Therefore, they provide converging evidence in support of the idea that grouping does not require attention, doing so with a different paradigm, with different stimuli, and measuring RTs, rather than accuracy. 

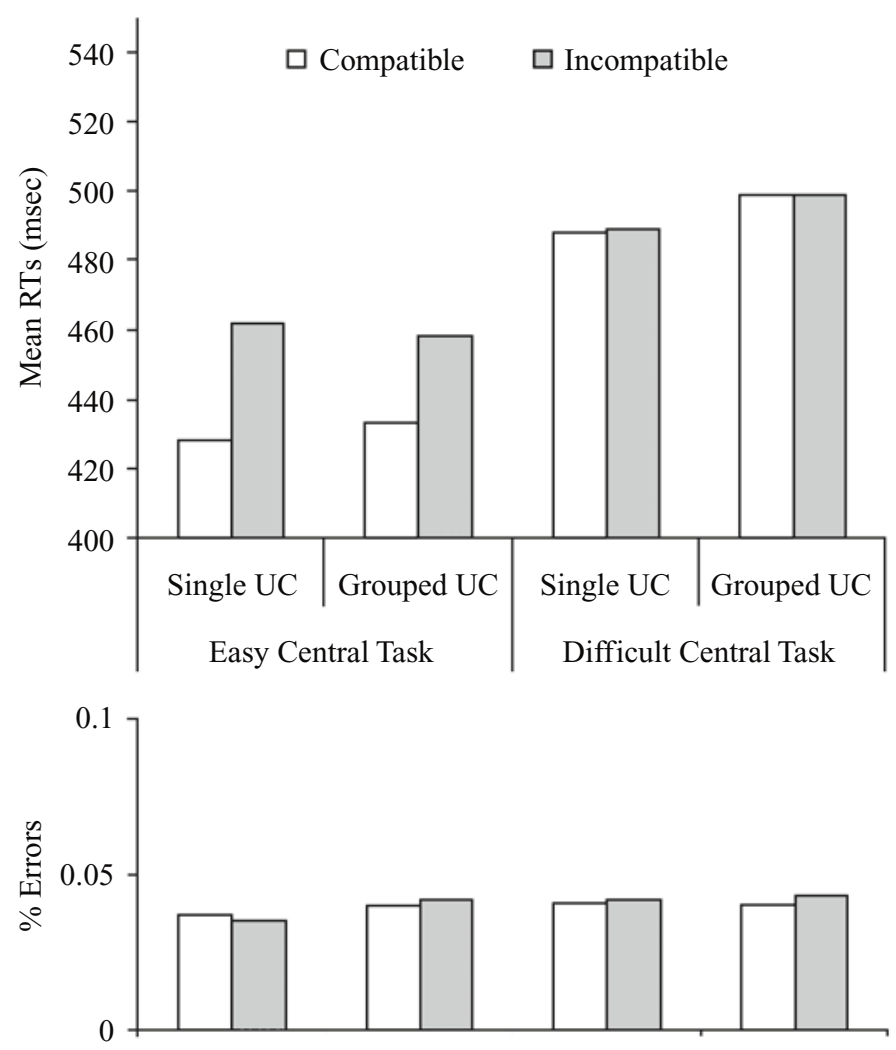

Figure 8. Results of Experiment 5. Mean reaction times (RTs, in milliseconds) as a function of the response elicited by the peripheral distractor (compatible vs. incompatible), type of distractor shape (single uniformly connected [UC] vs. grouped UC), and central task difficulty (easy vs. difficult). Central task difficulty was manipulated by varying target-distractor heterogeneity.

\section{GENERAL DISCUSSION}

The view, adopted by many theorists, that Gestalt grouping can occur without attention has been challenged by studies showing that subjects do not perceive even salient grouping patterns under conditions of inattention. Moore and Egeth (1997) showed that although grouping outside the focus of attention indeed cannot be the object of overt report, due perhaps to memory failure, its effects on visual perception can be observed when these are assessed using implicit, rather than explicit, measures. Since this finding constitutes the only piece of evidence to date suggesting that grouping occurs between object parts that lie entirely outside the focus of attention, it was important to ensure that it could be replicated when its potential problems were controlled for. The results of the present study confirm Moore and Egeth's claim that grouping does not require attention, with no possible concern that (1) attending to the target letter caused the surrounding perceptual group to be attended or that (2) occasional attentional leakages or (3) accrual of residual attentional resources to the periphery allowed grouping to occur.

In Experiment 1, we ensured that, under conditions of full attention, the Müller-Lyer illusion remained robust when the shafts and the arrowheads differed in color, although its magnitude decreased relative to the same-color condition, replicating Mukerji's (1957) early findings. Experiment 2 was a replication of Moore and Egeth's (1997, Experiment 3) illusion block, with the addition of a condition in which the attended lines and unattended dots differed in color. That is, it was similar to Experiment 1 but measured the strength of the illusion under conditions of inattention. The illusion was found to be significant in the different-color condition, although it was weaker than in the same-color condition. Experiment 3 was conducted in order to avoid a potential confound of grouping by proximity inherent in the Müller-Lyer stimuli and to seek converging evidence by employing another implicit measure of grouping (response compatibility effects; Eriksen \& Eriksen, 1974), with different stimuli and a different dependent measure (RT, in addition to accuracy). Responses to a central letter were influenced by whether, when present, a surrounding pattern made up of same-color elements elicited a compatible or an incompatible response, although this pattern was distant from the attended letter and had a different color from the target. Central task difficulty was manipulated in Experiments 4 and 5, in order to determine whether such compatibility effects might 
have resulted from attentional leakage to the periphery. Taken together, the results supported Moore and Egeth's claim that grouping does not require attention.

In Experiments 2-5, in contrast with the effects of grouping observed on the implicit measures, explicit measures revealed that the subjects had no memory of the unattended patterns. In this regard, it should be noted that our study was modeled after the illusion block used in Moore and Egeth's (1997) experiments, rather than on their inattention block (which used Mack et al.'s [1992] inattention procedure). This aspect of the present study may have biased our measure of explicit report. Because random- and pattern-matrix trials were randomly intermixed, the fact that the subjects failed to report seeing a pattern after the last experimental trial may have resulted from the fact that this last trial was sometimes a random-matrix trial. Thus, it remains possible that if all the subjects had been questioned immediately after a pattern-matrix trial about patterns appearing in the background, their performance on the explicit measure would have been above chance. To test this possibility, we examined whether being presented with a pattern-matrix display on the last experimental trial that immediately preceded the direct query increased the subjects' tendency to report seeing a pattern. In Experiment 2, 6 out of the 10 subjects who perceived no pattern at all had a pattern-matrix display on the last experimental trial, and 4 out of the 6 subjects who reported seeing a pattern had a random-matrix display on the last experimental trial. Out of these, only 1 of the 3 subjects who opted for the correct pattern in the forced choice query had a patternmatrix trial on her last experimental trial. In Experiment 3, 7 out of the 9 subjects had a pattern-matrix display on their last trial, yet none of them reported seeing a pattern. Finally, in Experiments 4 and 5, 4 out of 12 subjects and 6 out of 12 subjects, respectively, had a pattern-matrix trial as their last trial, yet only one of them reported seeing a pattern. On the basis of these observations, it seems safe to conclude that the subjects' failure to report on the peripheral pattern cannot be attributed to the fact that the last trial was not a pattern-matrix trial for all the subjects.

This replication of Moore and Egeth's (1997) dissociation between implicit and explicit measures of perception is important only because it further warns us against inferring about perceptual processes from subjects' explicit reports. Explicit reports may not provide an adequate measure of whether or not specific perceptual operations took place, for several reasons. First, the relevant perceptual information may be encoded in short-term memory and then forgotten. With shorter retention intervals, this information may be consciously perceived, but it may be rapidly erased and fail to be encoded in a memory store beyond iconic memory (see Lamme, 2003, on the relationship between awareness, attention, and memory). Alternatively, this information may be represented in the brain; that is, in the present instance, grouping processes may take place, as is reflected by their effects on subjects' responses, but may never reach awareness. Although the distinction between conscious and unconscious processes has become central to the study of perception in recent years and is at the heart of the quest for the neural correlates of consciousness (Crick \& Koch, 1998), it is important to underscore that this issue was not addressed in the present study. The data presented here did not allow us to determine whether attention is necessary for conscious perception or whether information has to be consciously perceived in order to affect behavior. Finally, we did not take the subjects' failure to report on the peripheral patterns to indicate that these were not attended. Instead, we resorted to direct manipulations of the amount of attention accruing to the peripheral patterns (Experiment 5).

Taken together, although the present results do not speak to the phenomenology of grouping perception that was the focus of the Gestaltist endeavor, they support the idea that the visual field can be organized into perceptual groups without attention.

\section{REFERENCES}

Abrams, R. A., \& Law, M. B. (2000). Object-based visual attention with endogenous orienting. Perception \& Psychophysics, 62, 818-833.

Baylis, G. C., \& Driver, J. (1992). Visual parsing and response competition: The effect of grouping factors. Perception \& Psychophysics, 51, 145-162.

BAYlis, G. C., \& Driver, J. (1993). Visual attention and objects: Evidence for hierarchical coding of location. Journal of Experimental Psychology: Human Perception \& Performance, 19, 451-470.

Behrmann, M., Zemel, R. S., \& Mozer, M. C. (1998). Object-based attention and occlusion: Evidence from normal participants and a computational model. Journal of Experimental Psychology: Human Perception \& Performance, 24, 1011-1036.

Ben-Av, M. B., SAGI, D., \& BraUn, J. (1992). Visual attention and perceptual grouping. Perception \& Psychophysics, 52, 277-294.

Crick, F., \& KoCH, C. (1998). Consciousness and neuroscience. Cerebral Cortex, 8, 97-107.

DeWAR, R. E. (1967). Stimulus determinants of the magnitude of the Mueller-Lyer illusion. Perceptual \& Motor Skills, 24, 708-710.

Driver, J., \& BAYLIS, G. C. (1989). Movement and visual attention: The spotlight metaphor breaks down. Journal of Experimental Psychology: Human Perception \& Performance, 15, 448-456.

DunCAN, J. (1984). Selective attention and the organization of visual information. Journal of Experimental Psychology: General, 113, 501517

Duncan, J., \& Humphreys, G. W. (1989). Visual search and stimulus similarity. Psychological Review, 96, 433-458.

Egeth, H. E., \& Lamy, D. (2003). Attention. In I. B. Weiner (Series Ed.) \& J. R. Graham \& J. A. Naglieri (Vol. Eds.), Comprehensive handbook of psychology: Vol. 4. Experimental psychology (pp. 269-292). New York: Wiley.

EgEth, H. E., \& Yantis, S. (1997). Visual attention: Control, representation and time course. Annual Review of Psychology, 48, 269-297.

Egly, R., Driver, J., \& Rafal, R. D. (1994). Shifting visual attention between objects and locations: Evidence from normal and parietal lesion subjects. Journal of Experimental Psychology: General, 123, 161-177.

Eriksen, B. A., \& EriKsen, C. W. (1974). Effects of noise letters upon the identification of a target letter in a nonsearch task. Perception \& Psychophysics, 16, 143-149.

Greene, E., \& Nelson, B. (1997). Evaluating Müller-Lyer effects using single fin-set configurations. Perception \& Psychophysics, 59, 293312 .

Handy, T. C., \& Mangun, G. R. (2000). Attention and spatial selection: Electrophysiological evidence for modulation by perceptual load. Perception \& Psychophysics, 62, 175-186.

Handy, T. C., Soltani, M., \& Mangun, G. R. (2001). Perceptual load and visuocortical processing: Event-related potentials reveal sensorylevel selection. Psychological Science, 12, 213-218.

Kramer, A. F., \& Jacobson, A. (1991). Perceptual organization and 
focused attention: The role of objects and proximity in visual processing. Perception \& Psychophysics, 50, 267-284.

Lamme, V. A. F. (2003). Why visual attention and awareness are different. Trends in Cognitive Sciences, 7, 12-18.

LAMY, D. (2000). Object-based selection under focused attention: A failure to replicate. Perception \& Psychophysics, 62, 1272-1279.

Lamy, D., \& EgETH, H. (2002). Object-based selection: The role of attentional shifts. Perception \& Psychophysics, 64, 52-66.

LAMY, D., \& TsaL, Y. (2000). Object features, object locations and object files: Which does selective attention activate and when? Journal of Experimental Psychology: Human Perception \& Performance, 26, $1387-1400$.

LamY, D., \& TsaL, Y. (2001). On the status of location in visual selective attention. European Journal of Psychology, 13, 305-342.

Lavie, N. (1995). Perceptual load as a necessary condition for selective attention. Journal of Experimental Psychology: Human Perception \& Performance, 21, 451-468.

Lavie, N., \& DE Fockert, J. W. (2003). Contrasting effects of sensory limits and capacity limits in visual selective attention. Perception \& Psychophysics, 65, 202-212.

LaVIE, N., \& Driver, J. (1996). On the spatial extent of attention in objectbased visual selection. Perception \& Psychophysics, 58, 1238-1251.

Lavie, N., \& Tsal, Y. (1994). Perceptual load as a major determinant of the locus of selection in visual attention. Perception \& Psychophysics, 56, 183-197.

Law, M. B., \& Abrams, R. A. (2002). Object-based selection within and beyond the focus of spatial attention. Perception \& Psychophysics, 64, 1017-1027.

MACK, A., \& Rock, I. (1998). Inattentional blindness. Cambridge, MA: MIT Press.

Mack, A., Tang, B., Tuma, R., Kahn, S., \& Rock, I. (1992). Perceptual organization and attention. Cognitive Psychology, 24, 475-501.

Moore, C. M., \& Egeth, H. E. (1997). Perception without attention: Evidence of grouping under conditions of inattention. Journal of Experimental Psychology: Human Perception \& Performance, 23, 339-352.

Moore, C. M., Yantis, S., \& Vaughan, B. (1998). Object-based visual selection: Evidence from perceptual completion. Psychological Science, 9, 104-110.

MountJoy, P. T. (1958). Effects of exposure time and intertrial interval upon decrement to the Müller-Lyer illusion. Journal of Experimental Psychology, 56, 97-102.

MUKERJI, N. (1957). Is "illusion" illusion? Journal of General Psychology, 57, 209-212.

NAVON, D. (1977). Forest before trees: The precedence of global features in visual perception. Cognitive Psychology, 9, 353-383.

NeISSER, U. (1967). Cognitive psychology. New York: Appleton-CenturyCrofts.

Norman, D. A., \& Bobrow, D. G. (1975). On data-limited and resourcelimited processes. Cognitive Psychology, 7, 44-64.

PALMER, S., \& Rock, I. (1994). Rethinking perceptual organization: The role of uniform connectedness. Psychonomic Bulletin \& Review, 1, 29-55.

Pollack, R. H. (1970). Magnitude of the Müller-Lyer illusion as a function of hue in the absence of lightness contrast. Proceedings of the Annual Convention of the American Psychological Association, 5, 53-54.

RATCLIFF, R. (1979). Group reaction time distributions and an analysis of distribution statistics. Psychological Bulletin, 86, 446-461.

Rock, I., Linnett, C. M., Grant, P., \& Mack, A. (1992). Perception without attention: Results of a new method. Cognitive Psychology, 24, 502-534.

Shomstein, S., \& Yantis, S. (2002). Object-based attention: Sensory modulation or priority setting? Perception \& Psychophysics, 64, 41-51.

Treisman, A., Kahneman, D., \& Burkell, J. (1983). Perceptual objects and the cost of filtering. Perception \& Psychophysics, 33, 527-532.
Treisman, A., \& Schmidt, H. (1982). Illusory conjunctions in the perception of objects. Cognitive Psychology, 14, 107-141.

VECERA, S. P. (1994). Grouped locations and object-based attention: Comment on Egly, Driver, and Rafal (1994). Journal of Experimental Psychology: General, 123, 316-320.

VeCERA, S. P., \& FARAH, M. J. (1994). Does visual attention select objects or locations? Journal of Experimental Psychology: General, 123, 146-160.

Watson, S. E., \& Kramer, A. F. (1999). Object-based visual selective attention and perceptual organization. Perception \& Psychophysics, 61, 31-49.

YANTIS, S. (2000). Goal-directed and stimulus-directed determinants of attentional control (tutorial). In S. Monsell \& J. Driver (Eds.), Control of cognitive processes: Attention and performance XVIII (pp. 73-104). Cambridge, MA: MIT Press.

\section{NOTES}

1. Lavie and Driver (1996, Experiment 4) have also argued that objectbased attention occurs only within attended regions, suggesting that object grouping requires attention. However, several failures to replicate this finding have been reported (Lamy, 2000; Law \& Abrams, 2002).

2. There were no different-length trials in the pattern-matrix condition in Moore and Egeth's (1997) experiment, but their experiment included the random-matrix condition, in which the lines differed in length on each trial. The present experiment included only pattern-matrix trials, in order to ensure that the subjects would pay attention to the arrowheads. However, it was important to include different-length trials in order to increase the subjects' belief that the lines could indeed have different lengths and, thus, ensure that they would comply with the task.

3 . Since random-matrix trials were neutral with respect to the response elicited by the task-relevant central letter, one would have expected responses in these trials to be slower than those in the compatible trials but faster than those in the incompatible condition. Yet they were slower than those in the incompatible condition. This unexpected finding might result from differences in spatial layout between the random- and the pattern-matrix conditions. First, the background was noisier in the random-matrix condition, since it contained several small perceptual groups (i.e., several clusters of colored squares), whereas in the patternmatrix condition the background contained only one colored perceptual group (the colored global letter). Moreover, in the random-matrix condition, colored squares were randomly scattered in the background, so that the minimum distance between the target and a colored square was $1^{\circ}$ of visual angle, whereas in the pattern matrix condition it was $3.5^{\circ}$. Thus, it may have been more difficult to filter out the irrelevant colored stimuli in the random- than in the pattern-matrix condition (Treisman et al., 1983). It should be noted, however, that this finding was not replicated in Experiments 4 and 5, in which the same conditions prevailed.

4. The data from Experiment 2 do not allow one to test this alternative account, because accuracy scores provide an all-or-none measure of performance on each trial.

5 . We thank an anonymous reviewer for this suggestion.

6. Including the 2 subjects who reported seeing the patterns in the background did not affect the pattern of results. That is, main effects of central task difficulty and compatibility were significant $[F(1,10)=$ $5.36, p<.05$, and $F(1,10)=8.25, p<.02$, respectively]; with no significant interaction between the two factors $[F(1,10)=1.26, p>.2]$.

7. Including the subject who reported seeing the patterns in the background did not affect the pattern of results. That is, the main effect of central task difficulty was significant $[F(1,10)=5.36, p<.05]$ and it interacted with compatibility, but there was no significant effect involving distractor shape type. Specifically, the decrease in compatibility effect was similar in the single-UC and the grouped-UC conditions $(F<1)$.

(Manuscript received October 21, 2003; revision accepted for publication March 3, 2005.) 